\title{
The Design of Centralized Heating Temperature Controller Based on MCU
}

\author{
Ding Shao ${ }^{1}$ \\ ${ }^{1}$ Student majoring in control engineering, North China Electric Power University,Baoding,Hebei,071003, China
}

\begin{abstract}
Combined with the practical project, based on the MCU technology and the sensor technology, this paper proposed the design proposal of the intelligent valve controller and the realization of the software and hardware. This design uses the PT100 platinum resistance as temperature sensor collecting the indoor environment. By controlling the heating electromagnetic valve, people can achieve the purpose of controlling the indoor temperature. At the same time through the key inputting, users can also set the comfortable temperature of heating and adjust the heating system of the start and stop time freely to achieve the purpose of energy conservation. This paper presents a new energy-saving idea and method from the angle of heating users. It has vast application prospect. Keywords: MCU, PT100, Intelligence, Heating, energy
\end{abstract}

\section{INTRODUCTION}

At present, ground heating system in north China area is not perfect. The heating system is relatively backward and most of the users' home have no intelligent temperature

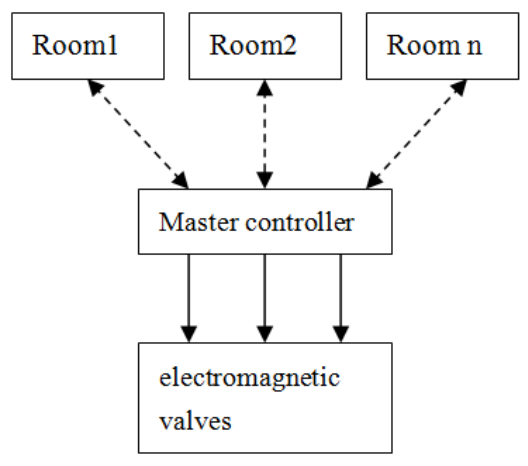

Figure 1 system diagram

\section{The hardware design of the intelligent temperature controller}

Intelligent temperature controller comprises temperature sensors, a main controller and electric valves. It can also measure a plurality of rooms. The temperature sensor is PT100, which has the advantages of high precision, good stability and strong reliability. The master controller is composed of MCU, liquid crystal display, clock chip and control device. The heating company's unreasonable fees for residents have also brought many disadvantages. For example, some home residents' heating temperature is so high that residents often open the window to cool down, which wastes a lot of energy; Some families have no one at home for a period of time, but still opening the heating as usual. It also wastes a lot of energy. This paper describes a design of intelligent temperature controller that users can control the heating time and heating temperature reasonably according to the actual needs of heating.

Through the Temperature sensor installed in each room, the intelligent controller collects each room temperature. The master controller receives the temperatureinformation and Process it. According to the requirements of temperature, the master controller can control the electromagnetic valves that are installed in the water separator on-off to complete control the heating, so as to realize the closed loop control of room temperature. The temperature sensor is integrated with a WIFI wireless communication module through which the temperature sensors can communication with the master controller.

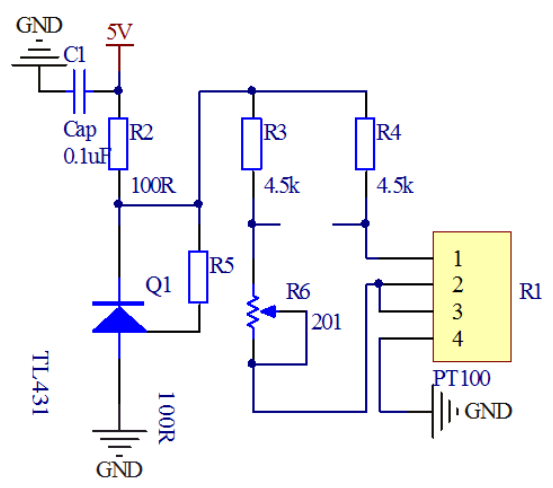

Figure 2 The sampling circuit

buttons. The structure diagram of intelligent temperature control system is shown in figure 1 .

\subsection{Design of Pt resistance temperature measurement} circuit

Platinum resistance (Pt100) is a kind of temperature sensor made of metal platinum $(\mathrm{Pt})$ whose resistance value can change with temperature. The nonlinearity of Pt100 is very small. In actual calculation, we generally use its linearization. According to the temperature 
characteristic of Pt100, this design uses three wire temperature measurement scheme. The sampling circuit is bridge measurement circuit (as shown in the figure 2). R3, R4, R6 and R1 form the temperature measuring bridge of the sensor. In order to guarantee the stability of the output voltage of the bridge, we use the TL431 to stable input voltage to $2.5 \mathrm{~V}$. The differential signal obtained from the bridge is very small, only a few millivolts, needed to be amplified by the amplifying circuit to the input MCU.

\subsection{Design of amplifying circuit}

Amplifying circuit adopts instrumentation amplifier circuit. This circuit can amplify weak signal, with the advantages of high gain, high input resistance and high common mode rejection ratio. Schematic diagram of the instrumentation amplifier is shown in Figure 3. It is composed of three operational amplifiers. Its gain is about 200 times. Amplified signal can be directly input STC12C5A60S2, a kind of MCU. This MCU have Road 8 and10 bit precision built-in ADC.

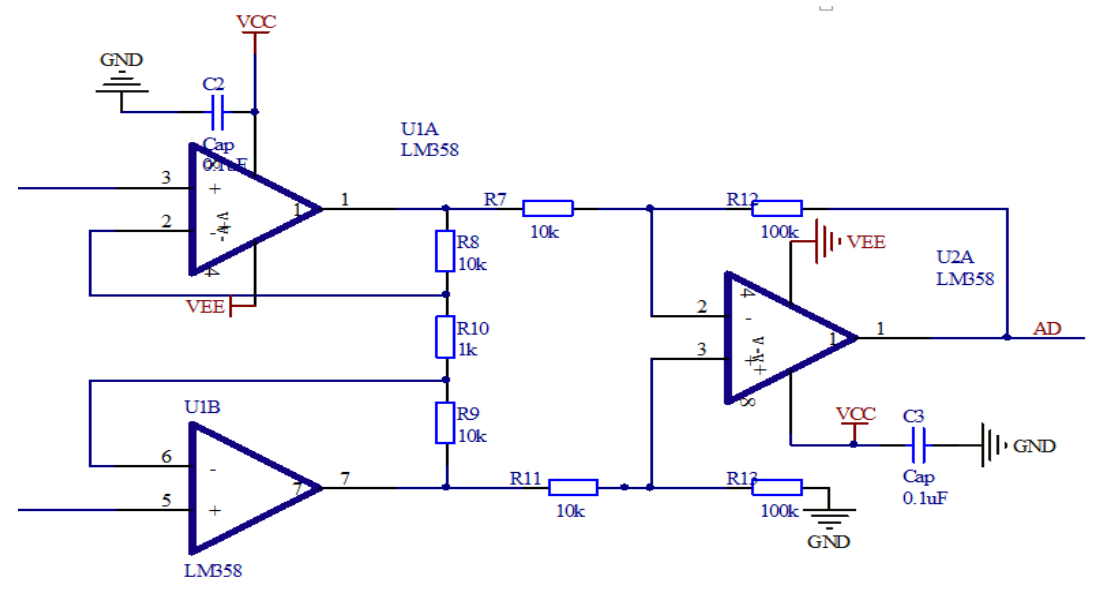

Figure 3 Schematic diagram of the instrumentation amplifier

\subsection{Design of WIFI module circuit}

Intelligent temperature controller uses URS-WIFI-232-T as the WIFI module. This module with low energy consumption and high degree of integration is very

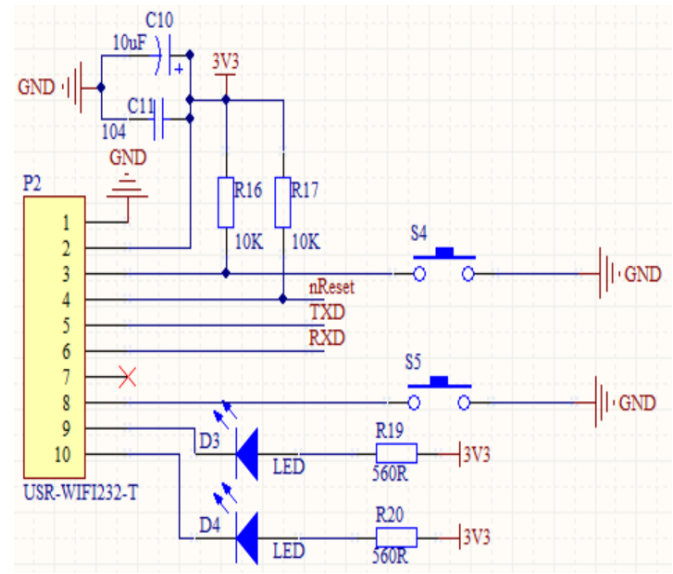

Figure 4 schematic circuit diagram of WIFI module conveniently and simply to use. This module connected with MCU can complete the communication between modules. Figure 4 is the schematic circuit diagram of the WIFI module.

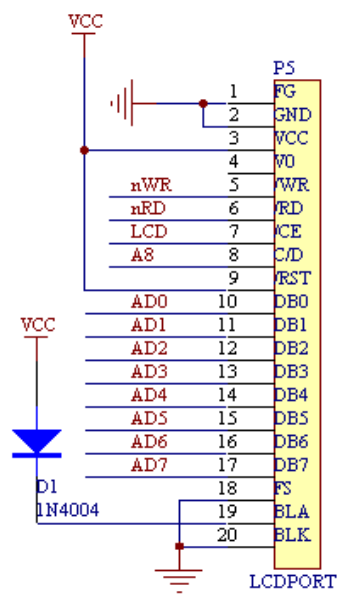

Figure 512864 modules 


\subsection{Design of the master controller}

The master controller of the intelligent temperature controller connected with WIFI module can communicate with 6 temperature sensors. At the same time, the liquid crystal display screen and keyboard of the master controller make users view each room and adjust timely.

The liquid crystal display screen is used by 12864 modules, which can be directly connected with the 51 MCU interface and can display several characters and graphics (as shown in figure 5).

\section{THE SOFTWARE DESIGN OF THE TEMPERATURE CONTROLLER}

Software plays a very important role in the system. The precise control of temperature relies on software program. According to the functions of the software program, the software program is generally divided into the main loop program, temperature acquisition program, data display program, real time clock program and communication program.

\subsection{The main loop program}

The main loop program of temperature controller is the most top-level programs. It mainly completes the initialization, reading parameter, temperature measurement and temperature displaying. After the instrument is power on, it will read the parameters that saved in the FLASH memory to initialize instrument. Then it will display the temperature. If any button is pressed, it will enter the program settings to set the program according to the definition of the function keys.

\subsection{The temperature calculation program}

According to the temperature characteristics of platinum resistance, we can be worth to the appropriate temperature by measuring the voltage at both ends of the platinum resistance. Voltage is a kind of analog signal. When we enter it into STC12C5A60S2, we can get its digital. Here is the form of the temperature and ADC.

\begin{tabular}{|lllllll|}
\multicolumn{1}{c}{} & \multicolumn{5}{c}{ Table1 } & The temperature calculation program \\
$\mathrm{ADC}$ & 188 & 180 & 172 & 164 & 156 & 148 \\
$\mathrm{~T}\left({ }^{\circ} \mathrm{C}\right)$ & 56.1 & 52.8 & 50.4 & 47.9 & 44 & 41.1 \\
$\mathrm{ADC}$ & 140 & 132 & 124 & 116 & 108 & 100 \\
$\mathrm{~T}\left({ }^{\circ} \mathrm{C}\right)$ & 37.7 & 34.6 & 31.2 & 28.2 & 24.7 & 21.5
\end{tabular}

Fitting chart line is shown below:

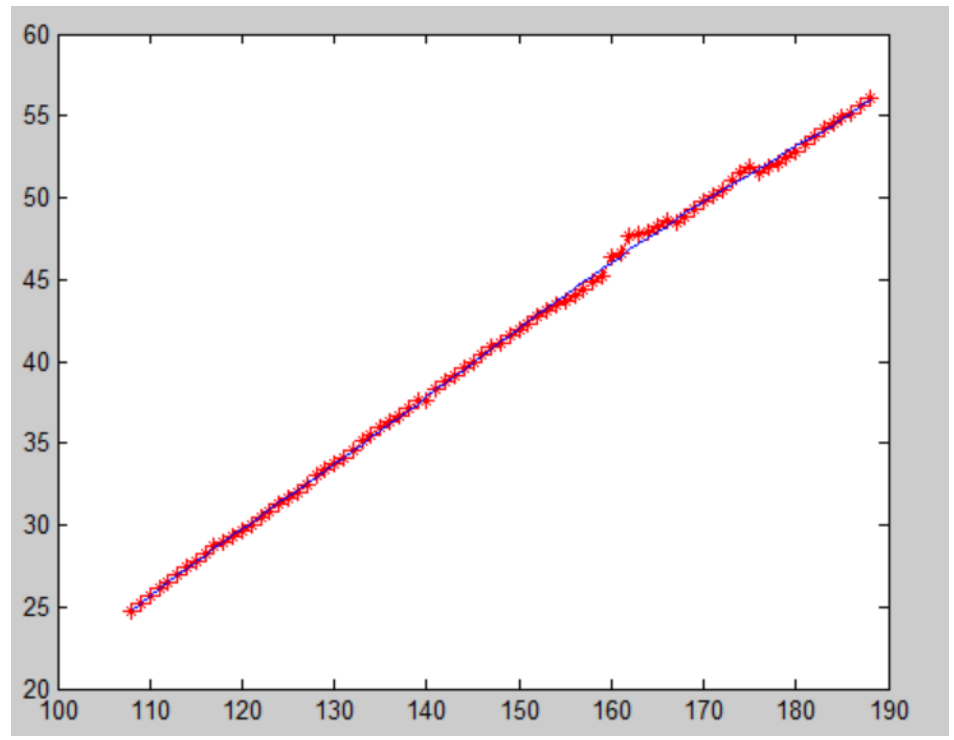

Figure 6 The temperature calculation program 
From the picture we can obviously find that the relationship between temperature and ADC is linear relationship. Relationship between temperature and ADC is

$\mathrm{ADC}=2.565 \mathrm{~T}+44.02$

\subsection{Real time clock program}

Real time clock counts seconds, minutes, hours, date of the month, month, day of the week, and year with leap year compensation valid up to 2100 . Here is the program $r$ of it.

void ds1302_init(void)

\{

$\mathrm{RST}=0$;

$\mathrm{SCK}=0$;

void ds1302_write_byte(uchar addr, uchar d)

\{ uchar i;

$\mathrm{RST}=1$;

addr $=$ addr \& $0 x F E$;

for $(i=0 ; i<8 ; i++)\{$

if (addr \& 0x01)

else $\{\mathrm{IO}=0 ;\}$ $\mathrm{IO}=1 ;\}$

$\mathrm{SCK}=1$;

$\mathrm{SCK}=0$;

addr $=$ addr $>>1 ;\}$

for $(\mathrm{i}=0 ; \mathrm{i}<8 ; \mathrm{i}++)\{$

if $(\mathrm{d} \& 0 \mathrm{x} 01)\{\mathrm{IO}=1 ;\}$

else $\{\mathrm{IO}=0 ;\}$

$\mathrm{SCK}=1$;

$\mathrm{SCK}=0$;

$d=d \gg 1 ;\}$

$\mathrm{RST}=0$;

\}

\section{THE HARDWARE DEBUGGING}

After the completion of welding intelligent temperature controller circuit, we can't rush to power. We must carry on the examination of circuit board carefully and use a multimeter to check whether there is weld. And then check the Schematic diagram to confirm that the welding and line is correct. Ensure that all lines are pathways. Check whether the model of components is correct and the package is appropriate. After all of the above completed, connect the power supply and check that the battery indicator on the LCD panel shows that the batteries are in good condition. If there is no abnormal phenomenon, it shows that the circuit is connected correctly. We can continue to operate.

\section{CONCLUSIONS}

The intelligent temperature controller solves the problem energy utilization of residents of North China heating in winter and has a very broad market space. In this paper, the main work includes:

1、 In design of the hardware circuit of analog part, according to the characteristics of the platinum resistance temperature sensor, we design three wire temperature measurement scheme and use the instrumentation amplifier circuit to amplify signal to achieve the measurement of temperature.

2、 Design liquid crystal display interface and wireless WIFI interface. It makes the instrument operation simple, convenient and flexible.

3、 In the design of the software, we realize the main loop program, A/D sampling program, data display program, temperature calculation program and realtime clock procedures and test it.

\section{ACKNOWLEDGEMENT}

The work was supported by College Students' Innovation and Entrepreneurship Training Plan of China under Grant No. 20142006.

\section{REFERENCES}

[1]Jiangbo Fan. Design of heating energy saving intelligent controller based on MCU .Journal of Harbin Vocational \& Technical College, 2012.

[2]Huijia Sun. Design and Realization of high-precision platinum resistance temperature measuring instrument. Hebei University, 2014.

[3] Jorgen Pihlflyckt, Control and Measurement System forMulti2chan2nelMicrowave Radiometer 【C/OL I.Russia: Helsinki University of Technology, 2007-02-12.

[4]Haisheng Gao. Simulation Electronic Technology Foundation. Jiangxi science and Technology Press. 287. 